\title{
RESISTENCIA A ACARICIDAS EN Rhipicephalus (Boophilus) microp/us DE ALGUNAS EXPLOTACIONES GANADERAS DE COLOMBIA
}

\section{ACARICIDAL RESISTANCE OF Rhipicephalus (Boophilus) microplus IN SOME COLOMBIAN CATTLE FARMS}

\author{
Alejandra Araque ${ }^{1}$, Sandra Ujueta ${ }^{2}$, Rodrigo Bonilla ${ }^{3}$, David Gómez ${ }^{4}$, Juan Rivera ${ }^{5}$
}

\begin{abstract}
${ }^{1}$ MVZ M.Sc. Farmacología. Docente Investigador, Facultad de Ciencias Pecuarias, Universidad de Ciencias Aplicadas y Ambientales, U.D.C.A calle 222 No. 57-33, Bogotá, D.C. Colombia. E-mail: aaraque@udca.edu.co ${ }^{2}$ MV M.Sc. Microbiología. Docente Investigador Facultad de Ciencias Pecuarias U.D.C.A. E-mail: sujueta@udca.edu.co ${ }^{3}$ MV. Director Técnico Ganadería. Laboratorios CARVAL de Colombia. E-mail: electrónico: rodrigo.bonilla@carval.com.co ${ }^{4}$ Estudiante MVZ Zootecnia, Facultad de Ciencias Agropecuarias, U.D.C.A, e-mail: david.leonardo.gomez@gmail.com ${ }^{5}$ Estudiante MVZ, U.D.C.A; e-mail: yususebastian@hotmail.com
\end{abstract}

Rev. U.D.C.A Act. \& Div. Cient. 17(1): 161-170, Enero-Junio, 2014

\section{RESUMEN}

El presente estudio correspondió a una evaluación de susceptibilidad de las garrapatas del ganado (Rhipicephalus (Boophilus) microplus) a dos acaricidas, en 71 explotaciones colombianas, ubicadas en diferentes regiones del país, con diferentes sistemas de producción y con una población bovina variable. Se recolectaron y se evaluaron muestras de la garrapata común del ganado, sometiéndolas a la prueba de resistencia recomendada por la FAO (Prueba de Inmersión de Adultas). De igual forma, se realizó una entrevista semiestructurada, donde se recolectó información para caracterizar las explotaciones, en aspectos de manejo de los acaricidas. Para tabular y analizar los datos, se empleó el programa de computación Excel de Microsoft Office (2010) y R-UCA, para recodificar las variables y generar gráficos de mortalidad y eficacia de los agentes acaricidas, etión y amitraz. Como resultado, se destaca que en el $97 \%$ de las explotaciones se presenta resistencia a el uso de amitraz y en el $52 \%$ al uso de etión, evidenciando la pérdida de la eficacia de este tipo de compuestos acaricidas, en el control de garrapatas en bovinos.

Palabras clave: Ectoparásitos, garrapatas, prueba Inmersión adultas (PIA), amitraz, etión, antiparasitarios.

\section{SUMMARY}

Problems related to parasitosis, such as ectoparasites, including ticks, are broad and important in veterinary practice due to increasing resistance of the indiscriminate use of acaricides. The present study corresponded to a survey on cattle tick (Rhipicephalus (Boophilus) microplus) susceptibility to two acaricides conducted in 71 ranches located in different Colombian regions, with different production systems and variable cattle population. Field samples of the cattle tick were collected and tested by the Adult Immersion Test recommended by FAO. Also, a semistructured interview was conducted, for collecting information to characterize some aspects of acaricide management in cattle ranches. The computer program Microsoft Office Excel (2010) was used to tabulate and analyze data, and R-UCA was used to recode variables and generate graphs of tick mortality and efficacy of the acaricides amitraz and ethion. It is highlighted that $97 \%$ and $52 \%$ of the ranches showed resistance to amitraz and ethion, respectively, evidencing a loss of efficacy by antiparasitic molecules to control cattle ticks.

Key words: Ectoparasites, adult immersion test (AIT), amitraz, ethion, antiparasitic drugs.

\section{INTRODUCCIÓN}

Los parásitos internos y externos del ganado continúan siendo una de las principales causas de pérdidas económicas en regiones tropicales, subtropicales y templadas del mundo (Pérez, 2003; Nari, 2005); Las afecciones parasitarias son consideradas como causa importante de déficits en la productividad ganadera, debido a la morbilidad y la mortalidad que provocan en los animales, alteraciones reproductivas y altos costos, ocasionados por su control, entre otros (Nari, 
2003). La garrapata Rhipicephalus (Boophilus) microplus (Canestrini, 1888), representa un desafío para los productores de ganado, ya que su ciclo de vida y amplia distribución en regiones tropicales y subtropicales favorecen el rápido desarrollo de resistencia a los acaricidas (Guerrero et al. 2012). Esta garrapata dura, de un solo hospedador, está presente en las zonas cálidas y bajas, aunque su adaptación es cada vez mayor a pisos térmicos más altos (Barker \& Murrell, 2004; Cortés et al. 2010).

Entre los efectos de la actividad patógena de esta garrapata, se encuentran la pérdida de peso, disminución en la producción de leche, daños en las pieles favoreciendo la aparición de miasis y la transmisión de agentes productores de enfermedades, como babesiosis y anaplasmosis (Linares, 2010), particularmente, en las regiones tropicales y subtropicales. Se ha estimado que el $80 \%$ de la población bovina del mundo está expuesta a infestación por garrapatas (FAO, 1984).

Una garrapata adulta ingurgitada provoca la pérdida, aproximadamente, de $1 \mathrm{~g}$ diario de peso vivo en el animal (Jonsson et al. 2006) y puede llegar a ingerir de 2 a $3 \mathrm{~mL}$ de sangre, durante toda su vida parasitaria (Betancourt \& Rodríguez, 2003), favoreciendo la presentación de alteraciones en la productividad y en la salud de bovinos. Las pérdidas económicas mundiales por garrapatas fueron estimadas en US\$7 billones y de esos, un billón de dólares corresponde a Latinoamérica (Castro et al. 2010); múltiples estudios en diversos países han evaluado el impacto de la infestación por garrapatas en la productividad bovina (FAO, 1984; Späth et al. 1994, 1994a).

La identificación de moléculas con propiedades acaricidas condujo rápidamente a la adopción de acaricidas químicos, como el método más usado para el control de garrapatas (Guerrero et al. 2012). Durante las últimas cuatro décadas, el desarrollo de productos garrapaticidas de gran eficacia y poder residual permitió al ganadero disponer de una herramienta de control práctica y adaptable, a diferentes sistemas de producción. Estas características, sumadas a una disminución de la toxicidad hacia los humanos en los más modernos grupos químicos, crearon un falso sentido de seguridad en el productor, quien sustituyó el diagnóstico y el asesoramiento profesional por la casi exclusiva utilización de fármacos (Díaz, 2012).

Los agentes acaricidas presentaron una evolución, tanto en el principio activo como en la vía de aplicación. Al comienzo, se realizaban exclusivamente por inmersión, actuando los principios activos por contacto directo y las formulaciones presentadas eran a base de organofosforados; luego, por su menor toxicidad y mayor eficacia, aparecen alternativas de tratamiento con piretroides sintéticos y diformamidinas. En la actualidad, el control se realiza con agentes de administra- ción parenteral o derrame dorsal (fipronilo, fluazuron, lactonas macrocíclicas, entre otros) (Cuore et al. 2008).

Muchos productores, frente al problema de disminución de eficiencia, cambian el producto sin criterio técnico, asesorado por el comercio: aumentan la dosis y disminuyen el intervalo entre baños, terminando con una frecuencia semanal y, únicamente, por último, recurren a un profesional veterinario, cuando la resistencia ya está instalada y sea más difícil su manejo (Cuore et al. 2012).

Es así, como la resistencia de diferentes especies de garrapatas a los productos garrapaticidas existe en la mayor parte de las regiones del mundo, donde se ha realizado control químico y es consecuencia de su uso inadecuado (Pereira et al. 2008).

El futuro de nuevos antiparasitarios, no sólo se encuentra comprometida por el progresivo aumento de los casos de resistencia y los crecientes costos de investigación y de desarrollo, sino también por cierta falta de conocimiento y de competencia para el descubrimiento de nuevas moléculas (Nari, 2003).

Los fallos operacionales, la lentitud y el escaso éxito de las campañas de erradicación de garrapatas que se observan en algunos países, como Australia, México, Puerto Rico, Uruguay, Cuba (FAO, 1989 b,c,d,e; Crom, 1992), confirman las dificultades que tienen los productores para acceder al uso de una estrategia que les permita mitigar o controlar el daño que causan las garrapatas a sus explotaciones.

La FAO ha incluido en sus recomendaciones el uso de las pruebas de inmersión de adultas (PIA), para las investigaciones de resistencia a los acaricidas, (FAO, 2004); sin embargo, existen otros métodos como la prueba del paquete de larvas y la inmersión de larvas (Baxter et al. 1999).

En Colombia, aun cuando no se han realizado estudios nacionales para determinar la extensión de la situación de resistencia, existen trabajos de carácter local o regional que visualizan el problema, encontrando que está presente en diversas regiones e, incluye, los acaricidas más utilizados (Díaz, 2012). Varios autores hallaron resistencia a organofosforados (OP) y piretroides sintéticos (PS), en departamentos, tales como Antioquia, Meta, Córdoba, Huila, Valle y Tolima (Betancourt, 1993; Benavides et al. 2000a, 2000b). Además, un estudio realizado en 1999, en los departamentos del Tolima, Huila y suroccidente de Cundinamarca, permitió conocer problemas de baja efectividad de acaricidas, en el $30 \%$ de los ganaderos de esta región, lo que obligaba a realizar baños garrapaticidas, con una alta frecuencia (Díaz et al. 2000). 
El presente estudio contempló el análisis de la situación de resistencia hacia dos moléculas acaricidas: amitraz y etión, de uso común en Colombia, para el control de la garrapata $R$. (B.) microplus, a partir del servicio de evaluación de eficacia de productos acaricidas ofrecido a productores y empresas farmacéuticas, a través de la PIA. También, incluyó el análisis descriptivo de los factores relativos al manejo de los acaricidas, mediante una entrevista semiestructurada.

\section{MATERIALES Y MÉTODOS}

La investigación, se desarrolló en el marco del Programa Universidad-Empresa, en el laboratorio de Microbiología en la U.D.C.A, que ofrece el servicio de evaluación in vitro de la eficacia de acaricidas usados en el control de $R$. (B.) microplus, identificadas según las claves taxonómicas de Caeiro (2006), para prestar asesoría en el control integral de garrapatas, bajo criterios técnicos y científicos.

El estudio fue de tipo transversal para la detección de resistencia a etión (organofosforado) y amitraz (diformamidina), en las poblaciones de garrapatas provenientes de las explotaciones que consultaron por la eficacia de estos productos. Las muestras incluidas en el estudio fueron seleccionadas por conveniencia, de acuerdo con las explotaciones de bovinos que solicitaron, entre enero de 2009 y diciembre de 2011, el servicio de asesoría técnica en el control de garrapatas. Las 71 explotaciones estudiadas cumplieron con los criterios de selección relacionados con número mínimo de 50 hembras adultas ingurgitadas (teleoginas) viables, inexistencia de tratamiento acaricida de contacto o endectocida por un periodo mínimo de un mes y encuesta, debidamente diligenciada, en el formato propuesto.

La recolección y el manejo de las muestras de garrapatas, se realizó según protocolos FAO $(1999,2004)$, orientando a los asistentes técnicos sobre la necesidad de recolectar hembras adultas ingurgitadas de la mayor cantidad de ganado, preferentemente, en horas de la mañana, de forma manual, para no causarles daño; almacenadas en envases limpios, debidamente identificados, con orificios de ventilación y una gasa humedecida. Se transportaron en el menor tiempo posible o incluyendo bolsas refrigerantes envueltas en papel para su transporte hasta el laboratorio. Las garrapatas ingurgitadas, viables y que no hubieran ovipositado, fueron seleccionadas y sometidas en el laboratorio a la prueba de inmersión de adultas (PIA), descrita por Drummond et al. (1973) y adaptada por Jonsson et al. (2007).

La prueba consistió en la inmersión a concentraciones comerciales indicadas en el manejo de los agentes acaricidas (208ppm, para amitraz y 664ppm, para etión), en relación con el efecto evidenciado en la postura y la viabilidad de los huevos. Las hembras ingurgitadas fueron lavadas con agua posterior a su colecta, para eliminar residuos de contaminantes que interfirieran con la prueba; cada acaricida fue probado con mínimo dos réplicas, 10 hembras por réplica. Cada réplica, se colocó en $20 \mathrm{~mL}$ de agua (control) o de $20 \mathrm{~mL}$ de cada uno de los acaricidas; permanecieron en la solución a una temperatura de $25^{\circ} \mathrm{C}$, por un periodo de 5 minutos, después fueron removidas, secadas con papel absorbente y colocadas en cajas de petri sobre cinta adhesiva, para incubación durante siete días, a temperatura entre 25 y $30^{\circ} \mathrm{C}$, con un fotoperiodo de 12 horas luz: 12 horas de oscuridad y humedad del $85-90^{\circ} \mathrm{C}$. El grupo control fue tratado únicamente con agua y mantenido bajo iguales condiciones de laboratorio (Drummond et al. 1973; Jonsson et al. 2007).

Posteriormente, se realizó el pesaje de la postura por producto y por controles, colocando las posturas en frascos con tapón de algodón, que se ubicaron verticalmente en incubación. La evaluación de la eclosión, se efectuó por medio de una lectura visual en términos de porcentaje, tomando el promedio de tres lecturas individuales.

A los siete días de incubación, se estimó el porcentaje de mortalidad a los 7 días del montaje de la prueba y la oviposición, utilizando las siguientes formulas (Álvarez et al. 2008):

$$
\text { Mortalidad }=\frac{\# \text { de garrapatas muertas }}{\sum \text { garrapatas }} \times 100
$$

Oviposición $=\frac{\# \text { de garrapatas tratadas que ovipositaron }}{\# \text { de garrapatas que ovipositaron en el control }} \times 100$

Se calculó la eficiencia reproductiva (ER), según cada acaricida y el control (Drummond et al. 1973):

$$
\mathrm{ER}=\frac{\text { Peso de los huevos }}{\text { Peso de las garrapatas }} * \% \text { Eclosión } * 20.000
$$

Se calculó la Eficacia del producto (EP), según cada acaricida (Abbot, 1925; Bravo et al. 2008):

$$
\mathrm{EP}=\frac{\text { ER control }- \text { ER del grupo tratado }}{\text { ER del grupo control }} * 100
$$

En todas las explotaciones del estudio, se aplicó una entrevista semiestructurada, para la captura de datos sobre la identificación y la ubicación, el manejo de los productos acaricidas (uso de indicaciones del producto, número de animales por bomba, frecuencia de baños, rotación de productos) y la percepción de la eficacia de los productos acaricidas. 
El estudio, de tipo transversal y descriptivo, contempló el análisis de las variables relativas a la resistencia de cada producto por población de garrapatas y explotación, ubicación de las explotaciones, manejo y percepción de los productos acaricidas con los programas estadísticos de Microsoft Excel 2010, STATGRAPHICS Plus y R-UCA (2008), (R D Core Team, 2009). También, se determinó la mediana (Me), medida de tendencia central, la cual, no es afectada por valores extremos (Dawson \& Trapp, 2002), que expresa un comportamiento de mayor exactitud respecto a la molécula estudiada, acompañado del Error Mediano (EM).

\section{RESULTADOS Y DISCUSIÓN}

Las muestras de garrapatas provinieron de 71 explotaciones de ganado bovino, ubicadas en cinco de las seis regiones naturales de Colombia; procedieron de fincas de la región Andina (73\%), de las regiones de la Orinoquia (10\%), región del Pacífico (10\%), región de la llanura del Caribe (4\%) y de la región de la Amazonía (3\%). De las muestras de la región Andina, 31\% procedieron del departamento de Santander, seguido por el departamento de Cundinamarca, 21\%; Antioquia, 15\%; Tolima, 13\%, con el 6\%, los departamentos de Quindío, de Caldas, de Huila y de Boyacá, apenas el 2\%.

La ubicación de las fincas de procedencia de las garrapatas coincide con la distribución de la ganadería nacional (Fedegan, 2006; Contexto Ganadero, 2013) y las condiciones de precipitación promedio mensual de 208,1 mm (Dprom $84,7 \mathrm{~mm}$ ), temperatura promedio $22,1^{\circ} \mathrm{C}$ y altitud promedio de 1140 msnm (Tabla 1) de las fincas, con la distribución reportada del ectoparásito en trabajos previos, en los que se ha encontrado en Colombia, entre 0 y 2600 msnm (Betancourt et al. 1992) y con probabilidad alta de hallar esta especie, en altitudes hasta 2.903 msnm (Cortés et al. 2010).

Tabla 1. Condiciones de precipitación, temperatura y altitud de los predios de los que proceden las muestras de garrapatas.

\begin{tabular}{|c|c|c|c|c|}
\hline $\begin{array}{c}\text { Precipitación } \\
\text { promedio/mes }\end{array}$ & $\begin{array}{c}\text { No. Explotaciones } \\
\mathbf{y}(\mathbf{\%})\end{array}$ & $\begin{array}{c}\text { Rango de temperatura } \\
\text { promedio }\left({ }^{\circ} \mathbf{C}\right)\end{array}$ & $\begin{array}{c}\text { Temperatura } \\
\text { promedio }\left({ }^{\circ} \mathbf{C}\right)\end{array}$ & msnm \\
\hline $45 \mathrm{~mm}$ & $5(7,0 \%)$ & $16,7-22,6$ & 19,4 & $1230-1900$ \\
\hline $135 \mathrm{~mm}$ & $30(42,3 \%)$ & $13,6-28,5$ & 21,4 & $10-2100$ \\
\hline $225 \mathrm{~mm}$ & $14(19,7 \%)$ & $19,2-28,5$ & 24,3 & $156-1550$ \\
\hline $315 \mathrm{~mm}$ & $20(28,2 \%)$ & $17,8-29,2$ & 22,6 & $10-1900$ \\
\hline $525 \mathrm{~mm}$ & $2(2,8 \%)$ & $16,5-20,1$ & 18,3 & $1460-2200$ \\
\hline Total & $71(100 \%)$ & & & \\
\hline
\end{tabular}

De acuerdo con el análisis de los datos obtenidos, a través de las entrevistas (71), 56\% de los productores perciben una aparente pérdida de eficacia de los agentes acaricidas que emplean en cada explotación, considerando el $46 \%$, de estos, que la actividad acaricida sobre el control de la garrapata es "regular" o parcialmente aceptable; $10 \%$, "mala" o deficiente, mientras que el $41 \%$, considera la actividad acaricida como aceptable o "buena" y, únicamente el 3\%, percibe que es "excelente", relacionada esta última, con una completo y esperado control de la garrapata en el animal.

Respecto al manejo de los productos, los ganaderos entrevistados usan bomba de espalda manual, con capacidad de 20L; el 70\%, realiza los baños según las indicaciones del laboratorio farmacéutico del producto; un 5\%, según indicaciones del asistente técnico y, un $25 \%$, procede con los baños según indicaciones propias, sumado al uso de concentraciones mayores a las indicadas o a la combinación de diferentes preparados acaricidas. El 41\% de las explotacio- nes bañan entre 4 y 6 animales por bomba; $44 \%$, entre 7 y $10 ; 10 \%$, entre 11-20 animales y, el 1\% de las explotaciones, llegan a bañar más de 20 animales por bomba. Teniendo en cuenta que cada bovino adulto debe ser bañado con 3 a 4L de la solución y la capacidad de la bomba, se observa que menos del $50 \%$ de las explotaciones que emplean la diformamidina y organofosforados, cumplen con esta indicación.

Respecto a los intervalos entre baños, el 6\% de las explotaciones realiza el baño cada 7 días; el 25\%, baña a los animales cada 8 a 15 días, con un promedio de 36 baños/año; el $12 \%$, cada 16-21 días, con un promedio de 16 baños/año; el $34 \%$, lo realiza cada 22 a 30 días, con un promedio de 12 baños/año; el 15\%, lo efectúa en un periodo mayor a 31 días y, el $8 \%$, realiza los baños según la necesidad de uso del agente acaricida, debido a la presencia del ectoparásito en los animales. El 7\% de productores cambian de grupo químico del acaricida cada 30 días; el 20\%, entre 31 y 60 días; el $12 \%$ lo cambian cada 61 a 120 días; el 26\%, en un 
tiempo mayor a 121 días; el $26 \%$ no ha cambiado de grupo hace más de un año y, el $9 \%$ de los productores, cuando el producto no funciona.

Se pudo deducir que los productores emplean diferentes estrategias de manejo de los productos acaricidas que coincide con su regular percepción sobre la eficacia de éstos y, posiblemente, en un intento por mejorar la eficacia de los mismos. Toman diversas medidas, como el incremento de la dosis o la frecuencia de aplicación, inclusive, hasta alcanzar límites tóxicos perjudiciales para el animal, práctica seguida por el remplazo arbitrario de grupos químicos y el correspondiente aumento en el costo de tratamientos. Buscan mejorar el control, aunque lo recomendado sea utilizar las dosis y las concentraciones adecuadas de acaricidas, para evitar, tanto la sub-dosificación como la sobredosificación en el ganado, ya que ambas producen resistencia (González et al. 2011).

Al momento de realizar el baño por aspersion es importante asegurar que el producto se dosifique correctamente, de acuerdo con el peso del animal, siguiendo las recomendaciones del laboratorio farmacéutico, para el correcto empleo de la formulación. Los animales, se tienen que saturar con el producto, especialmente, en la región axilar, zona inguinal y orejas, garantizando un buen cubrimiento del cuerpo, lo que se logra aplicando 1L de solución garrapaticida, por cada $100 \mathrm{~kg}$ de peso vivo, usando las boquillas adecuadas de los equipos de aplicación y sujetando, adecuadamente, el animal (Benavides et al. 2000b; Rodríguez et al. 2006).

Puesto que el desarrollo de resistencia en artrópodos depende de la frecuencia de aplicación de los insecticidas, así como de los ciclos de vida de los insectos (Rodríguez-Vivas et al. 2012), el manejo para retrasar la resistencia, incluye, la disminución en la frecuencia de aplicaciones, a través de todo el año. En el mismo sentido, Nari (2003) menciona que para garrapatas en Colombia, las fincas que realizaban más de 12 aplicaciones al año, en particular de piretroides sintéticos, poseían un riesgo dos veces mayor de presentar resistencia, que las que cumplían menos de este número de aplicaciones al año y Jonsson et al. (2000) encontraron, para amitraz, que más de cinco aplicaciones por año, incrementan las probabilidades de desarrollo de resistencia.

El uso racional de antiparasitarios de uso externo, incluye no sólo la disminución en la frecuencia de aplicaciones, a través de todo el año, sino la rotación de productos, ya que aparentemente no existe resistencia cruzada entre organofosforados y diformamidinas, lo cual, que retrasa el desarrollo de resistencia (Alonso et al. 2006).

\section{Prueba de inmersión de adultas (PIA).}

Mortalidad. De acuerdo con el criterio de interpretación de los resultados (eficacia $>90 \%$ ) (FAO, 2004), se identificó que, únicamente el $1 \%$ de las muestras provenientes de las explotaciones incluidas en el estudio, presentaron una mortalidad entre 91 y 100\%, para amitraz (Me 30\%) y el 13\%, para etión (Me: 55\%). En la tabla 2, se muestran los porcentajes de mortalidad agrupados por tratamiento y por rango, donde se observa una mayor mortalidad para amitraz, en los rangos inferiores, a diferencia del etión, mayor mortalidad, en los rangos superiores. El control negativo no superó el rango de 0-10\% de mortalidad, asegurando así la correcta manipulación del material biológico (Gallardo \& Morales, 1999).

Tabla 2. Porcentaje de predios por rango de mortalidad y por tratamiento, mediante la prueba de inmersión de adultas (PIA).

\begin{tabular}{|c|c|c|c|}
\hline \multirow{2}{*}{$\begin{array}{c}\text { Rango de mortalidad } \\
(\%)\end{array}$} & Amitraz & Etión & Control \\
\cline { 2 - 4 } & $6 \%$ & $0 \%$ & $69 \%$ \\
\hline $0,1-10 \%$ & $15 \%$ & $8 \%$ & $31 \%$ \\
\hline $11-20 \%$ & $17 \%$ & $13 \%$ & $0 \%$ \\
\hline $21-30 \%$ & $20 \%$ & $4 \%$ & $0 \%$ \\
\hline $31-40 \%$ & $17 \%$ & $13 \%$ & $0 \%$ \\
\hline $41-50 \%$ & $6 \%$ & $10 \%$ & $0 \%$ \\
\hline $51-60 \%$ & $8 \%$ & $10 \%$ & $0 \%$ \\
\hline $61-70 \%$ & $4 \%$ & $11 \%$ & $0 \%$ \\
\hline $71-80 \%$ & $6 \%$ & $10 \%$ & $0 \%$ \\
\hline $81-90 \%$ & $0 \%$ & $8 \%$ & $0 \%$ \\
\hline $91-100 \%$ & $1 \%$ & $13 \%$ & $0 \%$ \\
\hline
\end{tabular}


El amitraz que fue utilizado en forma masiva como alternativa de control a principios de los años 90, a causa de la dispersión de la resistencia de $R$. (B.) microplus a los piretroides y, posteriormente, dentro de los programas intensivos para el control del ectoparásito en algunas explotaciones (Soberanes et al. 2002), mostró en el estudio baja mortalidad. Esto es debido al uso intensivo e indiscriminado de la molécula, ocasionando una fuerte presión de selección, que elimina los individuos susceptibles, convirtiéndose así el manejo de los productos, en el principal agente de selección.

En general, los resultados obtenidos durante el estudio muestran baja eficacia de los acaricidas, siendo estos re- sultados no esperados para el amitraz (mortalidad $<30 \%$ ) y etión (mortalidad $<55 \%$ ), puesto que se esperarían porcentajes aceptables, mínimo del 90\%, considerando el mecanismo de acción de estos principios activos, encargados de afectar directamente el estadio adulto de la garrapata (Petrie et al. 2006; Nari, 2005).

Oviposición. En las garrapatas tratadas, se observó un mejor comportamiento del etión respecto a la oviposición, $24 \%$ de las explotaciones, en un rango entre 61 y $100 \%$, mientras que para el amitraz, el porcentaje de oviposición fue mayor, $70 \%$ de las explotaciones, entre 61 y $100 \%$. Los valores de porcentaje de oviposición de los huevos de las garrapatas por tratamiento y rango, se presentan en la tabla 3.

Tabla 3. Porcentaje de predios por rango de oviposicion y por tratamiento, mediante la prueba de inmersión de adultas (PIA).

\begin{tabular}{|c|c|c|c|}
\hline \multirow{2}{*}{$\begin{array}{c}\text { Rango de oviposición } \\
\text { (\%) }\end{array}$} & \multicolumn{3}{|c|}{ Tratamiento } \\
\cline { 2 - 4 } & Amitraz & Etión & Control \\
\hline $0 \%$ & $0 \%$ & $11,3 \%$ & $0 \%$ \\
\hline $0.1-10 \%$ & $1,4 \%$ & $4,2 \%$ & $0 \%$ \\
\hline $11-20 \%$ & $1,4 \%$ & $14,1 \%$ & $0 \%$ \\
\hline $21-30 \%$ & $4,2 \%$ & $21,1 \%$ & $0 \%$ \\
\hline $31-40 \%$ & $8,5 \%$ & $8,5 \%$ & $0 \%$ \\
\hline $41-50 \%$ & $7 \%$ & $4,2 \%$ & $0 \%$ \\
\hline $51-60 \%$ & $7 \%$ & $12,7 \%$ & $0 \%$ \\
\hline $61-70 \%$ & $14,1 \%$ & $8,5 \%$ & $0 \%$ \\
\hline $71-80 \%$ & $18,3 \%$ & $5,6 \%$ & $0 \%$ \\
\hline $81-90 \%$ & $16,9 \%$ & $7 \%$ & $0 \%$ \\
\hline $91-100 \%$ & $21,1 \%$ & $2,8 \%$ & $100 \%$ \\
\hline
\end{tabular}

Era de esperar que el porcentaje de oviposición encontrado para el amitraz fuera mucho menor que el observado en el estudio, debido a que la molécula tiene la capacidad de inhibir la oviposición en las hembras ingurgitadas, interfiriendo con su ciclo evolutivo en particular (Bravo et al. 2008); además, incluye otros mecanismos de acción que afectan el proceso de oviposición y la eclosión, tales como la acción de la octopamina, neuromodulador involucrado en el comportamiento reproductivo de los artrópodos, por actividad sobre receptores específicos en el oviducto, lo cual, potencia la acción letal de la molécula (Alonso et al. 2006; Botana et al. 2002).

Eclosión. Porcentajes de eclosión de los huevos de las garrapatas, mayores al 51\%, se observaron en el $74,5 \%$ de las garrapatas tratadas con amitraz y, en el 16,9\%, de las explotaciones tratadas con etión. Los valores de porcentaje de eclosión de los huevos de las garrapatas por tratamiento y por rango, se presentan en la tabla 4.

Los datos obtenidos durante el estudio muestran que el porcentaje de eclosión es elevado para los huevos de las garrapatas tratadas con amitraz, puesto que al haber sido expuestos al tratamiento se esperaría que no se presentara oviposición o que los huevos no mostraran viabilidad para eclosionar, demostrando así efecto acaricida.

Eficacia. Del total de explotaciones incluidas en el estudio, únicamente para el 4,2\% de las muestras de garrapatas tratadas con amitraz y para el $56 \%$ de las muestras de garrapatas tratadas con etión, los acaricidas mostraron una eficacia de producto superior al $90 \%$, es decir, que solamente en tres explotaciones incluidas en el estudio, el amitraz mostró efectividad en el control de las garrapatas y, en 40 explota- 
ciones, para el etión. En dos explotaciones (2,8\%), se observó eficacia simultánea de los dos productos amitraz y etión, confirmándose así, la pérdida de eficacia de los productos estudiados, sobre las poblaciones de garrapatas analizadas.

Tabla 4. Porcentaje de predios por rango de eclosión y por tratamiento, mediante la prueba de inmersión de adultas (PIA).

\begin{tabular}{|c|c|c|c|}
\hline \multirow{2}{*}{$\begin{array}{c}\text { Rango de eclosión } \\
\text { (\%) }\end{array}$} & \multicolumn{3}{|c|}{ Tratamiento } \\
\cline { 2 - 4 } & Amitraz & Etión & Control \\
\hline $0 \%$ & $0 \%$ & $18,3 \%$ & $0 \%$ \\
\hline $0,1-10 \%$ & $1,4 \%$ & $18.3 \%$ & $0 \%$ \\
\hline $11-20 \%$ & $1,4 \%$ & $11,3 \%$ & $0 \%$ \\
\hline $21-30 \%$ & $4,2 \%$ & $4,2 \%$ & $0 \%$ \\
\hline $31-40 \%$ & $8,5 \%$ & $16,9 \%$ & $0 \%$ \\
\hline $41-50 \%$ & $9,9 \%$ & $14,1 \%$ & $1,4 \%$ \\
\hline $51-60 \%$ & $23,9 \%$ & $4,2 \%$ & $1,4 \%$ \\
\hline $61-70 \%$ & $21,1 \%$ & $9,9 \%$ & $11,3 \%$ \\
\hline $71-80 \%$ & $22,5 \%$ & $2,8 \%$ & $45,1 \%$ \\
\hline $81-90 \%$ & $7 \%$ & $0 \%$ & $38 \%$ \\
\hline $91-100 \%$ & $0 \%$ & $0 \%$ & $2,8 \%$ \\
\hline
\end{tabular}

Para clasificar como eficaz a un garrapaticida debe tener un rango mayor a $90 \%$ de eficacia, nivel minimo aceptado internacionalmente por la FAO (Bravo et al. 2008). Considerando la evaluación de los productos acaricidas sobre las garrapatas por explotación, aunque se evidencia un mejor comportamiento del etión sobre el amitraz, los resultados son preocupantes. Requieren el diseño de un plan estrategico orientado al uso racional y acorde con la situación particular, respecto al desarrollo de resistencia en cada población de garrapatas. Los valores de eficacia de producto (EP) para cada tratamiento y rango, se presentan en la tabla 5.

Tabla 5. Porcentaje de predios por rango de eficacia y por tratamiento, mediante la prueba de inmersión de adultas (PIA).

\begin{tabular}{|c|c|c|}
\hline \multirow{2}{*}{ Rango de eficacia (\%) } & \multicolumn{2}{|c|}{ Tratamiento } \\
\cline { 2 - 3 } & Amitraz & Etión \\
\hline $0 \%$ & $0 \%$ & $0 \%$ \\
\hline $0.1-10 \%$ & $1,4 \%$ & $0 \%$ \\
\hline $11-20 \%$ & $8,5 \%$ & $0 \%$ \\
\hline $21-30 \%$ & $4,2 \%$ & $0 \%$ \\
\hline $31-40 \%$ & $19,7 \%$ & $0 \%$ \\
\hline $41-50 \%$ & $14,1 \%$ & $5,6 \%$ \\
\hline $51-60 \%$ & $11,3 \%$ & $1,4 \%$ \\
\hline $61-70 \%$ & $19,7 \%$ & $9,9 \%$ \\
\hline $71-80 \%$ & $9,9 \%$ & $11,3 \%$ \\
\hline $81-90 \%$ & $7 \%$ & $15,5 \%$ \\
\hline $91-95 \%$ & $1 \%$ & $8 \%$ \\
\hline $96-100 \%$ & $3 \%$ & $48 \%$ \\
\hline
\end{tabular}


De acuerdo con la prueba de inmersión de adultas y los parámetros analizados, mortalidad, porcentaje de oviposición, porcentaje de eclosión y eficacia de producto, se confirma la percepción en campo de los productores sobre la falta de eficacia de productos acaricidas, tras su uso indiscriminado y cotidiano. Se demuestra la pérdida considerable de eficacia de las dos moléculas, amitraz y etión, para las explotaciones analizadas, manteniéndose para algunas de las explotaciones (56\%), el etión como opción acaricida, pero descartando el uso del amitraz, para el control de poblaciones de garrapatas en la mayoría (97\%) de las explotaciones.

El fenómeno de resistencia en poblaciones de garrapatas aparece paulatinamente como resultado del uso indiscriminado y errado de las moléculas en campo (FAO, 2004).

De acuerdo con lo anterior, se hace imperativo generar estrategias que conlleven al uso racional de antiparasitarios, apoyadas en el uso del laboratorio para el diagnóstico de eficacia y en la observación, análisis sistemático y seguimiento de la situación en campo, como herramienta pertinente y oportuna al alcance del productor ganadero, para orientar el uso adecuado y particular de las moléculas en su explotación, que redundará en la disminución del desarrollo de resistencia, en poblaciones de garrapatas.

Agradecimientos: Al laboratorio de microbiología de la Universidad de Ciencias Aplicadas y Ambientales U.D.C.A; a laboratorios CARVAL de Colombia; A María Teresa Ríos y John Jairo Gómez, por el apoyo estadístico. Conflicto de intereses: El presente trabajo fue preparado con la participación de todos los autores, quienes declaramos que no existe conflicto de intereses que ponga en riesgo la validez de los resultados o la voluntad de publicación de los mismos.

\section{BIBLIOGRAFÍA}

1. ABBOTT, W. 1925. A method of computing the effectiveness of an insecticide. J. Econ. Entomol. 18:256257.

2. ALONSO, A.; RODRÍGUEZ, I.; FRAGOSO, H. 2006. Resistencia de la garrapata Boophilus microplus a los ixodicidas. Arch. Med. Vet. UACh. 38(2):105-113.

3. ÁlVAREZ, V.; LOAIZA, J.; BONILLA, R.; BARRIOS, M. 2008. Control in vitro de garrapatas (Boophilus microplus; Acari: Ixodidae) mediante extractos vegetales. Rev. Biol. Trop. 56(1):291-302.

4. BARKER, S.C.; MURRELL, A. 2004. Systematics and evolution of ticks with a list of valid genus and species names. Parasitology. 129:S15-36.
5. BAXTER, G.; GREEN, P.; STUTTEN, M.; BARKER, S. 1999. Detecting resistance to organophosphates and carbamates in the cattle tick, Boophilus microplus, with a propoxur based chemical test. Exp. Appl. Acarol. 23:907-914.

6. BENAVIDES, E.; ROMERO, N.; RODRÍGUEZ, J. 2000a. Situación actual de resistencia de la garrapata Boophilus microplus a acaricidas en Colombia. Segunda entrega Carta Fedegan. 60:13-18.

7. BENAVIDES, E.; ROMERO, N.; RODRÍGUEZ, J. 2000b. Situación actual de la resistencia de la garrapata Boophilus microplus a acaricidas en Colombia. Recomendaciones de manejo integrado. Carta Fedegan. 61:14-24.

8. BETANCOURT, J.A.; GARCÍA, O.; ROQUEME, L.; NAVARRETE, M. 1992. Distribución y niveles de infestación por garrapatas en bovinos de Córdoba, noroeste de Sucre y noroeste de Antioquia. Revista ICA. 27:63-76.

9. BETANCOURT, A. 1993. Susceptibilidad de varias cepas de la garrapata Boophilus microplus a diferentes acaricidas. Rev. Cebú. 22:53-55.

10. BETANCOURT, J.; RODRÍGUEZ, C. 2003. Estudios sobre identificación y control de garrapatas de bovinos en el departamento de Cauca. Rev. Regional Novedades Tecnicas. 3:38-43.

11. BOTANA, L.; LANDONI, F.; JIMÉNEZ, G. 2002. Farmacología y Terapéutica Veterinaria. U. Santiago de Compostela, España: Mc Graw Hill Interam. 715p.

12. BRAVO, M.; CORONADO, A.; HENRÍQUEZ, H. 2008. Eficacia in vitro del amitraz sobre poblaciones Boophilus microplus provenientes de explotaciones lecheras del estado Lara, Venezuela. Zoot. Trop.. 26(1):35-40.

13. CAEIRO, V. 2006. Reflexão sobre a taxonomia actual dos Ixodidae. A sistemática morfológica versussistemática molecular - o género Rhipicephalus e o género Boophilus. Rev. Portug. Cienc. Vet. 101(557558):37-39.

14. CASTRO, J.; RIFRAN, G.; PIAGGIO, G.; SCHUMAKER, T. 2010. Rhipicephalus (Boophilus) microplus (Acari: Ixodidae) resistance to Fipronil in Uruguay evaluated by in vitro bioassays. Vet Parasitol. 7:169-172. 
15. CONTEXTO GANADERO. 2013. Listado con la distribución del inventario ganadero en el país. Disponible desde Internet en: http://contextoganadero.com/ ganaderia-sostenible/presentan-nuevo-listado-conla-distribucion-del-inventario-ganadero-en-el-pais (con acceso 06/11/2013).

16. CORTÉS, J.; BETANCOURT, J.; ARGÜELLES, J.; PULIDO, L. 2010. Distribución de garrapatas Rhipicephalus (Boophilus) microplus en bovinos y fincas del Altiplano cundiboyacense (Colombia). Rev. Corpoica: Ciencia y Tecnología Agropec. 11(1):73-84.

17. CROM, R. 1992. Eradication of Boophilus microplus (Acari: Ixodidae) in Puerto Rico. Ann. NY Acad. Sci. 653:64-71

18. CUORE, U.; ALTUNA, M.; CICERO, L.; FERNÁNDEZ, F.; LUENGO, L.; MENDOZA, R.; TRELLES, A. 2012. Aplicación del tratamiento generacional de la garrapata en la erradicación de una población multirresistente de Rhipicephalus (Boophilus) microplus en Uruguay. Rev. Vet. (Montevideo). 48(187):5-13.

19. CUORE, U.; CARDOZO, H.; TRELLES, A.; NARI, A.; SOLARI, M. 2008. Características de los garrapaticidas utilizados en Uruguay. Eficacia y poder residual. Rev. Vet. (Montevideo). 43(169):13-24.

20. DAWSON, B.; TRAPP, R.G. 2002.. Bioestadística Médica. $3^{\text {a }}$ ed. México, D.F. Editorial El Manual Moderno. 367p.

21. DÍAZ, E. 2012. Mecanismos moleculares y bioquímicos de resistencia a acaricidas en la garrapata común de los bovinos Rhipicephalus microplus. Rev. Col. Cienc. Animal. 5(1):72-81.

22. DÍAZ, E.; BENAVIDES, E.; PARRA, H.; ARCOS, C.; RIVEROS, E.; JARAMILLO, F.; LONDOÑO, E. 2000. Investigación epidemiológica de las principales limitantes parasitarias en explotaciones ganaderas del Tolima, Huila y suroccidente de Cundinamarca. Informe final proyecto PRONATTA-CORPOICA.

23. DRUMMOND, R.; ERNST, S.; TREVINO, J.; GLADNEY, W.; GRAHAM, O. 1973. Boophilus annulatus and Boophilus microplus: Lab. Test of Insecticides. J. Econ. Entomol. 66:130-133.

24. FAO. 1984. Ticks and tick borne disease control. A practical field manual. Rome, I Tick Control. 299p.
25. FAO. 1989a. Economics of tick eradication in Puerto Rico. Animal Production and Health Paper. Rome. 75:91-93.

26. FAO. 1989b. History of tick eradication in Puerto Rico. Animal Production and Health Paper. Rome; Module 1: Ticks 71:8-15.

27. FAO. 1989c. The campaign against Boophilus spp. in Mexico: benefits, problems and prospects. An. Prod. Health Paper; Rome. 25:16-27.

28. FAO. 1989d. History of tick eradication in Uruguay. An. Prod. Health Paper; Rome. 75:45-59.

29. FAO. 1989e. History of tick control and eradication in the Republic of Cuba. An. Prod. Health Paper; Rome. 75:60-73.

30. FAO. 1999. Resistance of ecto- and endo-parasite: current and future solution, 67th General sessión. Int. Comité. OIE. Paris. p.17-21.

31. FAO. 2004. Guideline resistance management and integrated parasite control in ruminants. Acaricide resistance: diagnosis, management and prevention Agr. Dept. Animal Production and Health Division. Roma, Italia. p.25-77.

32. FEDERACIÓN COLOMBIANA DE GANADEROS FEDEGAN-. 2006. Plan estratégico de la ganadería colombiana 2019. Por una ganadería moderna y solidaria. Ed. Sanmartín Obregón y Cía. Colombia. 296p.

33. GALLARDO, J.S.; MORALES, J. 1999. Boophilus microplus (Acari: Ixodidae): Preoviposición, ovoposición, incubación de los huevos y geotropismo. Bioagro. 11(3):77-87.

34. GONZÁLEZ, A.; TAPIAS, D.; PÉREZ, M.; CARVAJALINO, M.; VELANDIA, D.; BORGES, R. 2011. Evaluación de Acaricidas para el control de garrapatas (Riphicephalus (Boophilus) microplus) que afectan al ganado bovino de doble propósito usando modelos lineales generalizados. Rev. Fac. Agr. LUZ. 28:487502.

35. GUERRERO, F.; LOVIS, L.; MARTINS, J. 2012. Acaricide resistance mechanisms in Rhipicephalus (Boophilus) microplus. Rev. Bras. Parasit. Vet. 21(1):1-6.

36. JONSSON, N.N.; MAYER, D.G.; GREEN, P.E. 2000. Possible risk factor on Queensland dairy farms for acari- 
cide resistance in cattle tick (Boophilus microplus). Vet. Parasitol. 88:79-92.

37. JONSSON, N.; MILLER, R.; ROBERTSON, J. 2007. Critical evaluation of the modified-adult immersion test with discriminating dose bioassay for Boophilus microplus using American and Australian isolates. Vet. Parasitol. 146:307-313.

38. JONSSON, N.; HOLDSWORTH, P.; KEMP, D.; GREEN, P.; PETER, R.; BRUIN, C.; VERCRUYSSE, J. 2006. World Association for the Advancement of Veterinary Parasitology (W.A.A.V.P.) guidelines for evaluating the efficacy of acaricides against ticks (Ixodidae) on ruminants. Vet. Parasitol. 136(1):29-43.

39. LINARES VILLALBA, S.E. 2010. Manejo integral de las garrapatas una propuesta eficiente y sostenible con el medio ambiente. Agronomía, 16(2):13-21.

40. NARI, A. 2003. Resistencia a los Antiparasitarios: Estado actual con énfasis en América Latina. Estudio FAO.

41. NARI, A. 2005. Estado actual de la resistencia de Boophilus microplus en America Latina y el Caribe. Perspectivas de aplicación del control integrado. Roma, Italia: FAO.

42. PEREIRA, M.C.; LABRUNA, M.B.; SZABÓ, M.P.; KLAFKE, G.M. 2008. Rhipicephalus (Boophilus) microplus. Biologia, controle e resistência. $1^{\text {a }}$. Ed. São Paulo MedVet Livros. vol. 1. 169p.

43. PÉREZ, C. 2003. Revisión. Consideraciones sobre el manejo de la resistencia y control integrado de la garrapata (Boophilus microplus). Disponible desde Internet en: http://www.mgap.gub.uy/DGSG/InformacionTecnica/Garrapata/RevisionManejodeResistenciayCIG.pdf (con acceso 14/02/2012).
44. PETRIE, A.; WATSON, P. 2006. Statistics for Veterinary and Animal Science ( ${ }^{\text {nd }}$ Ed.). lowa, USA: Blackwell Publishing. 299p.

45. R DEVELOPMENT CORE TEAM (R D Core Team), 2009. $R$ : A language and environment for statistical computing. R Foundation for Statistical Computing, Vienna, Austria. Disponible desde Internet en: http:// www.R-project.org (con acceso 14/02/2012).

46. RODRÍGUEZ, R.; ALONSO, M.; RODRÍGUEZ, F.; FRAGOSO, H.; SANTAMARIA, V.; ROSARIO, R. 2006. Prevalence and potencial risk factors for oganophosphate and pyrethroid resistance in Boophilus microplus ticks on cattle ranches from the State of Yucatan, México. Vet. Parasitol. 136:335-342.

47. RODRÍGUEZ-VIVAS, R.; HODGKINSON, J.; TREES A. 2012. Resistencia a los acaricidas en Rhipicephalus (Boophilus) microplus: situación actual y mecanismos de resistencia. Rev Mex. Cienc. Pec. 3(Supl 1):9-24.

48. SOBERANES, N.; SANTAMARIA, M.; FRAGOSO, H.; GARCÍA, Z. 2002. Primer caso de resistencia al Amitraz en la garrapata del ganado Boophilus microplus en México. Rev. Técn. Pec. 40(1):81-92.

49. SPÄTH, E.; GUGLIELMONE, A.; SIGNERINI, A.; MANGOLD, A. 1994. Estimación de las pérdidas económicas directas producidas por la garrapata $\mathrm{BoO}$ philus microplus y las enfermedades asociadas en la Argentina. Primera Parte. Vet. Rural. 23:341-360.

50. SPÄTH, E.; GUGLIELMONE, A.; SIGNERINI, A.; MANGOLD, A. 1994a. Estimaciónde las pérdidas económicas directas producidas por la garrapata Boophilus microplus y las enfermedades asociadas en la Argentina. Segunda Parte. Vet. Rural. 23:389-396.

Recibido: Marzo 21 de 2013

Aceptado: Abril 28 de 2014

\section{Como Citar:}

Araque, A.; Ujueta, S.; Bonilla, R.; Gómez, D.; Rivera, J. 2014. Resistencia a acaricidas en Rhipicephalus (Boophilus) microplus de algunas explotaciones ganaderas de Colombia. Rev. U.D.C.A Act. \& Div. Cient. 17(1):161-170. 